Rev. Int. Contam. Ambie. 34 (1) 7-21, 2018

DOI: 10.20937/RICA.2018.34.01.01

\title{
PLAGUICIDAS AGRICOLAS: UN MARCO DE REFERENCIA PARA EVALUAR RIESGOS A LA SALUD EN COMUNIDADES RURALES EN EL ESTADO DE SONORA, MÉXICO
}

\author{
María Isabel SILVEIRA-GRAMONT ${ }^{1}$, María Lourdes ALDANA-MADRID ${ }^{1}$ *, \\ Julián PIRI-SANTANA ${ }^{2}$, Ana Isabel VALENZUELA-QUINTANAR ${ }^{3}$, \\ ${\text { Graciela JASA-SILVEIRA }{ }^{4} \text { y Guillermo RODRÍGUEZ-OLIBARRIA }}^{1}$
}

${ }^{1}$ Departamento de Investigación y Posgrado en Alimentos, Universidad de Sonora. Rosales y Bulevar Luis Encinas, Centro, Hermosillo, Sonora, México, C. P. 83000

${ }^{2}$ Departamento de Ciencias Químico Biológicas, Universidad de Sonora. Rosales y Bulevar Luis Encinas, Centro, Hermosillo, Sonora. C. P. 83000

${ }^{3}$ Centro de Investigación en Alimentación y Desarrollo, A.C. Carretera a la Victoria, kilómetro 0.6, Hermosillo, Sonora, México, C. P. 83304

${ }^{4}$ Human Rights Research and Education Centre, University of Ottawa. 57 Louis Pasteur, Ottawa, Ontario, Canadá, K1N 6N5

*Autor para correspondencia: lourdes.aldana@unison.mx

(Recibido abril 2016; aceptado mayo 2017)

Palabras clave: agroquímicos, exposición pasiva, dispersión de residuos, localidades rurales

\section{RESUMEN}

En diferentes proyectos realizados en Sonora, México, se comprobó la presencia de contaminación por agroquímicos en trabajadores y habitantes de localidades rurales aledañas a campos de cultivo. Para evaluar el peligro que representa para la salud de los habitantes la exposición pasiva de dichos compuestos, se requiere información confiable y actualizada sobre cultivos sembrados y plagas, así como cantidades, dosis y formas de aplicación de los plaguicidas utilizados, especialmente aquellos con potencial de riesgo para la salud. En este trabajo se propone un marco de referencia que permita identificar la información con que se cuenta a nivel local, como lo es el Distrito de Desarrollo Rural (DDR) 144-Hermosillo sobre cultivos y sus plagas, además de la intensidad y frecuencia de aplicación de plaguicidas por aspersión. En el periodo 2010 a 2014, nueve cultivos ocupaban el $80 \%$ de la superficie sembrada en el DDR 144 Hermosillo y podían emplear 24 productos agroquímicos aplicados por aspersión. De éstos, los insecticidas organofosforados y herbicidas llegan a aplicarse de 16 a $211 \mathrm{t}$ en 72500 ha en una temporada, varios de estos plaguicidas son moderadamente persistentes en el ambiente y se consideran con efectos adversos a la salud por organizaciones nacionales e internacionales. Se concluye que en los predios agrícolas se asperjan suficientes cantidades de plaguicidas peligrosos, que pueden afectar la salud de los habitantes de localidades cercanas.

Key words: agrochemicals, passive exposition, residue dispersion, rural communities 


\begin{abstract}
Different research projects in Sonora, Mexico, have proven contamination from pesticides in workers and locals living in communities near agricultural fields. To evaluate the health hazards from passive exposition of their inhabitants to such compounds, it is required reliable and updated information on the crops sown, their pests, as well as pesticide amounts, doses and forms of application, especially of agrochemicals that pose health risk potential. This work proposes a reference frame to identify the required information at a local level such as the Rural Development District (RDD) 144 Hermosillo, on crops, pests, besides the intensity and frequency of the application of pesticides by spraying. In the period from 2010 to 2014 , nine crops occupied 72500 ha $(80 \%)$ of the sown area in RDD Hermosillo, and could employ 24 most frequent agrochemical products applied by spray. Of those organophosphate insecticides and herbicides, 16 and $211 \mathrm{t}$ were applied over 72500 ha in one season. Several of these pesticides are moderately persistent in the environment and they are considered with adverse health effects by national and international organizations. It is concluded that in agricultural fields, enough quantities of hazardous pesticides are sprayed, which might possibly affect the health of inhabitants in the nearby communities.
\end{abstract}

\section{INTRODUCCIÓN}

La agricultura es una actividad importante en Sonora, México, por su aporte a la alimentación nacional y su contribución a la economía del estado a través de la exportación de la mayoría de sus productos agrícolas. En los valles agrícolas Sonora es donde se localiza gran parte de la producción agrícola, la cual es principalmente de riego y de tipo comercial. La búsqueda de una mayor eficiencia en la producción conlleva el uso de grandes cantidades de plaguicidas que se aplican para reducir pérdidas ocasionadas por microorganismos, hongos, insectos, malezas y otros depredadores de los cultivos. La mayoría de los plaguicidas son productos tóxicos que ocasionan daños al ambiente y a los seres vivos. En la actualidad la mayoría de los científicos coinciden en que hay un exceso de aplicación de plaguicidas en el sector agrícola, lo que ha dado lugar al deterioro de las tierras de cultivo y a la generación de resistencia para algunas plagas (Oerke 2006, Harsimran y Harsh 2014, Albert 2015). Por otra parte, las políticas de desarrollo rural en México ponen énfasis en aumentar la productividad y rentabilidad de los cultivos, lo que en cierta forma favorece el aumento en el uso de estos plaguicidas (SAGARPA 2015a).

Hasta el momento no se cuenta con cifras actualizadas de la cantidad y frecuencia de su aplicación, tampoco existen datos estadísticos y/o gubernamentales actualizados sobre su uso con especificación de cultivo, principio activo, forma de aplicación y dosis recomendadas.
Muchos de los plaguicidas que se usan en la agricultura se aplican por aspersión de polvos o mezclas acuosas al follaje de las plantas y/o malezas que crecen junto a los cultivos. Estos se dispersan en el ambiente y afectan la salud de los trabajadores agrícolas que no utilizan equipo de protección, se acumulan en los suelos y aguas superficiales y son transportados por el aire a otros sitios en función de las condiciones atmosféricas. Los plaguicidas aplicados por fumigaciones aéreas pueden ser arrastrados por el viento a varios kilómetros de distancia del área donde se aplican (Fritz et al. 2011, Butler-Ellis et al. 2016). Además, otras sustancias en la formulación pueden tener potencial tóxico y acarrear un riesgo adicional a humanos y al ambiente (Harsimran y Harsh 2014).

Varias investigaciones realizadas en diferentes regiones han demostrado en forma consistente que la dispersión de plaguicidas hacia localidades cercanas a campos de cultivo deposita niveles elevados de estos compuestos tanto en el aire, como en el polvo de calles, patios y viviendas (Quandt et al. 2006, Ward et al. 2006, Moreno-Villa et al. 2012, Wofford et al. 2014, Damalas 2015). Adicionalmente, se ha comprobado que el riesgo de exposición en el ambiente por la dispersión de estos compuestos depende principalmente del tipo y cantidad de plaguicida asperjado, la altura del cultivo y la forma de aplicación, así como las condiciones atmosféricas, los equipos de aspersión y la velocidad con que se aplica (USEPA 1997, Hewitt 2002, Nuyttens et al. 2010, Fritz et al. 2011, Butler-Ellis et al. 2016).

Respecto al impacto que los residuos de plaguicidas causan sobre la salud de los seres humanos, 
el riesgo dependerá en gran medida de la magnitud y frecuencia de la exposición, de la toxicidad de la sustancia, así como de la vulnerabilidad de subgrupos poblacionales, principalmente niños y mujeres, a dichos compuestos. El impacto en la salud en población no expuesta ocupacionalmente a plaguicidas resulta por la exposición crónica a pequeñas cantidades de estos compuestos. Estos efectos se denominan de "largo plazo", ya que pueden tardar años en manifestarse clínicamente. Tal es el caso de cáncer, efectos neurotóxicos, endócrinos, de la reproducción y otros (Koureas et al. 2012, Damalas 2015, Ross et al. 2015, Teedon et al. 2015, Butler-Dawson et al. 2016).

De aquí se puede inferir la importancia de contar con datos sobre superficies cultivadas, tipo y épocas de cultivos. Además de contar con información puntual sobre uso de plaguicidas dañinos para la salud $\mathrm{y} / \mathrm{o}$ que se aplican con mayor frecuencia e intensidad.

No se encuentran disponibles estadísticas oficiales sobre el uso agrícola de plaguicidas en México, excepto las generales de censos o encuestas, donde se registran las cantidades de fertilizantes, insecticidas y plaguicidas agrícolas (INEGI 2016). Sin embargo, a nivel local y estatal es posible obtener datos más específicos sobre plagas y plaguicidas de los distritos de desarrollo rural (DDR) de la Secretaría de Agricultura, Ganadería, Desarrollo Rural, Pesca y Alimentación (SAGARPA), a través de sus diferentes dependencias y de los sistemas de información agrícola. Asimismo, en cada estado los productores están agrupados en asociaciones que comparten información sobre diversos aspectos de importancia para la productividad de sus cultivos y que se difunden a través de internet.

Es por ello que este estudio se circunscribe a zonas agrícolas de riego y agricultura comercial, donde se aplican plaguicidas de forma intensiva para mantener la rentabilidad de los cultivos. En el estado de Sonora, México, se seleccionó al DDR 144 Hermosillo, dado que las investigaciones recientes sobre salud y plaguicidas en las comunidades agrícolas, se han realizado en su mayoría en él.

En Sonora, México, se han realizado estudios de monitoreo de plaguicidas a través de diferentes investigaciones en suelo y agua (Cantu et al. 2011, Moreno-Villa et al. 2012, Leal et al. 2014), en alimentos (Aldana et al. 2008a, Aldana et al. 2008b, Aldana et al. 2011) y en matrices biológicas de habitantes y trabajadores de zonas agrícolas (Cejudo et al. 2012, Huen et al. 2012, Meza et al. 2012, Ochoa et al. 2012, Aldana et al. 2013, Meza et al. 2013). La mayoría de los sujetos de estudio de residuos en matrices biológicas fueron trabajadores agrícolas, por lo que no se conoce el efecto del grado de exposición pasivo de residentes de las localidades rurales aledañas a los campos de cultivo. De investigaciones anteriores realizadas por este grupo (Silveira et al. 2011, Ochoa et al. 2012, Aldana et al. 2013), se estimó que la contaminación por plaguicidas ocurre en un porcentaje mayor a $50 \%$ en las personas que trabajan y habitan en localidades aledañas a los campos agrícolas (CA) y que un factor de riesgo asociado con la presencia de residuos de plaguicidas es la cercanía de la vivienda a dichos campos (Silveira et al. 2011).

Aunque el uso de plaguicidas cerca de un lugar no significa que, necesariamente, los individuos que lo habitan están expuestos, su frecuente aplicación y su persistencia química pueden aumentar la probabilidad de exposición y tener implicaciones en el deterioro de la salud a largo plazo (Ross et al. 2015).

El objetivo de este estudio fue proponer una metodología para planear iniciativas que minimicen la exposición potencial a plaguicidas en comunidades que habitan cerca de campos de cultivo, muchas de las cuales se han construido sobre tierras agrícolas cultivadas en el pasado.

\section{MATERIALES Y MÉTODOS}

En este trabajo se propuso un marco metodológico de referencia para estimar el potencial de exposición pasiva a plaguicidas asperjados de los habitantes de localidades aledañas a campos de cultivo. Éste consistió en analizar la información de fuentes secundarias sobre cultivos, superficies sembradas y plagas que presentan dichos cultivos. Además de plaguicidas recomendados para su control a partir de la información con que cuenta un DDR, la que proporciona el gobierno federal y estatal sobre éste, la bibliografía científica relevante y los manuales técnicos de la SAGARPA para la zona.

\section{Sitio de estudio}

El DDR 144 Hermosillo está integrado por tres municipios: Hermosillo, San Miguel de Horcasitas y Carbó. El clima en esta región agrícola varía de seco a muy seco con temperaturas mínimas de $15^{\circ} \mathrm{C}$ en los meses de invierno y máximas entre $37{ }^{\circ} \mathrm{C}-48^{\circ} \mathrm{C}$ en los meses de verano, con precipitaciones entre 75 y $250 \mathrm{~mm}$ entre junio y agosto. El área agrícola de este distrito es predominantemente de riego, con agricultura comercial en aproximadamente 94000 ha (INEGI 2014), aunque la superficie cultivada varía año con año, dependiendo de la disponibilidad de agua y de los cultivos anuales que se siembren. En 
la figura 1 se muestran los cultivos con mayor superficie de siembra en los últimos cinco años (2010 a 2014).

Las localidades rurales aledañas a los campos de cultivo están generalmente pobladas por trabajadores, jornaleros agrícolas y sus familias, lo que ocasiona que sus habitantes estén expuestos a la contaminación proveniente de los agroquímicos empleados en dichos campos, así como por las limitaciones de infraestructura y vivienda (Silveira et al. 2016).

El censo de localidades para el DDR 144 Hermosillo del 2010 (INEGI 2010) reporta 310 localidades con más de 100 hab de las cuales $90 \%$ están a poca distancia de campos agrícolas. En ellas hay un $14 \%$ de viviendas sin agua entubada, un $33 \%$ sin drenaje y un $15 \%$ con piso de tierra, lo cual es indicativo de una infraestructura que facilita la contaminación por plaguicidas que puede llegar a través del aire, de las vestimentas de trabajadores agrícolas y de la aplicación domiciliaria de plaguicidas (Quandt et al. 2006, Fenske et al. 2013, Remoundou et al. 2014).

Particularmente en el municipio de Hermosillo, hay alrededor de 40 localidades rurales de más de 100 habitantes (100-30 000) rodeadas de campos agrícolas (varias a menos de $500 \mathrm{~m}$ ), con un grado bajo de infraestructura urbana (agua, drenaje, electricidad, viviendas, calles de tierra) (SEDESOL 2015). Es importante señalar que en estas localidades o cerca de ellas hay escuelas rurales (primarias y secundarias) que también pueden recibir los efectos de la dispersión de plaguicidas desde los campos cercanos.

\section{Fuentes de información secundaria}

Para describir las características de la producción agrícola del DDR 144 Hermosillo, se obtuvo información bibliográfica del número y tipo de cultivos que hay en el distrito. Se consultó la base de datos del Sistema de Información Agrícola y Pecuaria (SIAP 2015) de la SAGARPA, el Anuario Estadístico del estado de Sonora 2014 (INEGI 2014) y escritos técnicos agrícolas sobre los cultivos predominantes en el distrito. Se resumió la información estadística con datos de las superficies sembradas de cultivos del DDR144 Hermosillo, Sonora, México, de los últimos cinco años (2010-2014). Se incluyó una descripción general de todos los cultivos, en particular, de los nueve que ocupaban $80 \%$ de la superficie de siembra (alfalfa, calabaza, garbanzo, naranja, nogal, sandía, sorgo, trigo y vid).

En lo que se refiere a plagas más frecuentes y plaguicidas indicados para su control en el DDR144 Hermosillo, se consultaron las guías técnicas de manejo de cultivos del Instituto Nacional de Investigaciones Forestales, Agrícolas y Pecuarias (INIFAP 2010) de la SAGARPA, así como bibliografía publicada por organismos y especialistas del sector agrícola de Sonora. Esta información se limitó a los cultivos de garbanzo, trigo y vid (Cuadro I). El listado de plaguicidas agrícolas contenía información sobre plaguicidas que se aplican a cada cultivo, los cuales incluyen ingredientes activos (IA) y productos comerciales (PC) que los contienen, presentaciones, dosis recomendadas, plagas que combaten y uso agrícola (SENASICA 2011; Cuadro II).

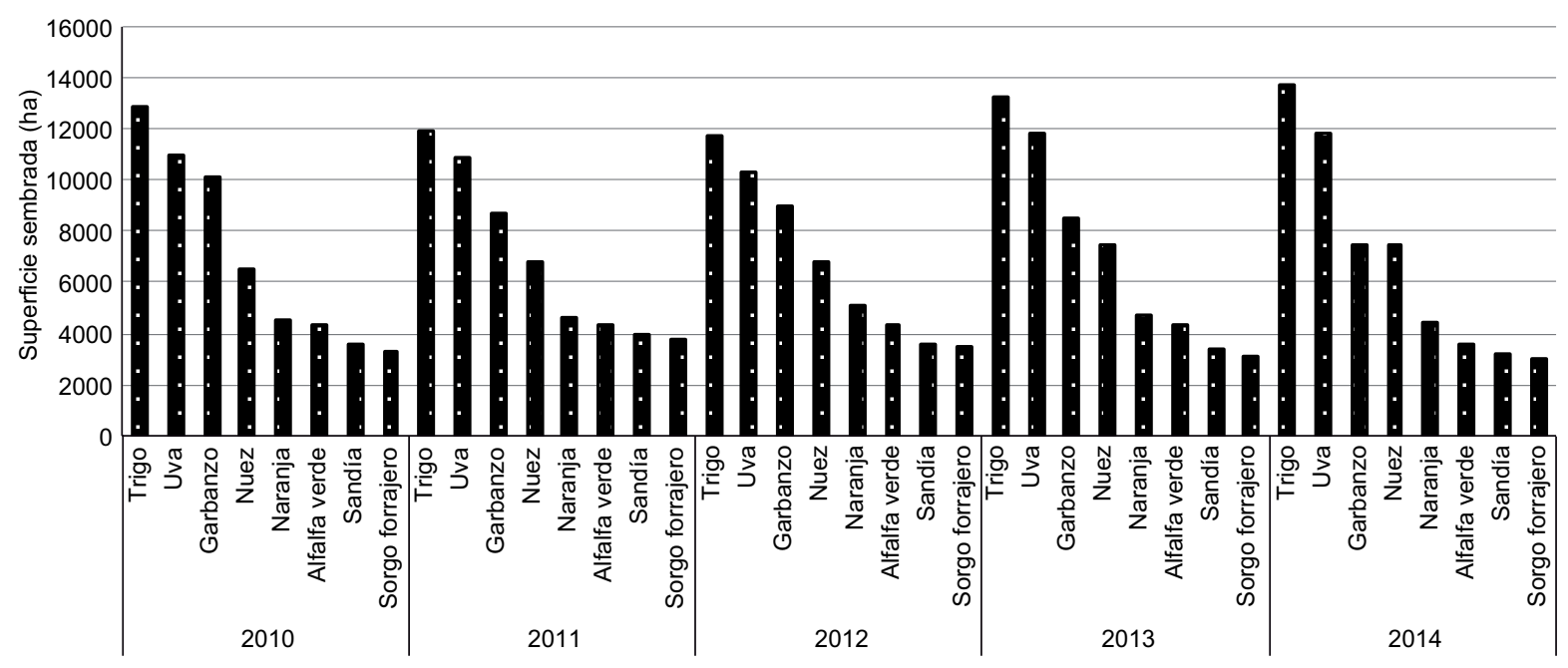

Fig. 1. Superficie sembrada de cultivos de mayor superficie agrícola en el Distrito de Desarrollo Rural 144 de Hermosillo de 2010 a 2014

Fuente: SIAP (2015) 
CUADRO I. INFORMACIÓN DE LA BASE DE DATOS DE PLAGUICIDAS DE USO AGRÍCOLA PUBLICADO POR EL SERVICIO NACIONAL DE SANIDAD, INOCUIDAD Y CALIDAD AGROALIMENTARIA

\begin{tabular}{|c|c|}
\hline Columna & Descripción \\
\hline Cultivo & $\begin{array}{l}\text { Alfalfa, calabaza, garban- } \\
\text { zo, naranjo, nogal, sandia, } \\
\text { sorgo, trigo, vid }\end{array}$ \\
\hline Empresas & $\begin{array}{l}\text { Bayer de México, S.A. de } \\
\text { C.V., Monsanto Comercial, } \\
\text { S.A. de C.V., Syngenta } \\
\text { Agro, S.A. de C.V., Helm } \\
\text { de México, S.A. }\end{array}$ \\
\hline Ingrediente activo & $\begin{array}{l}\text { Nombre del ingrediente } \\
\text { activo }\end{array}$ \\
\hline Condiciones de uso & Libre, restringido \\
\hline Nombre comercial & Varios \\
\hline Presentación & $\begin{array}{l}\text { Polvo humectable, polvo, } \\
\text { solución acuosa, concen- } \\
\text { trado emulsionable, polvo } \\
\text { soluble, micro encapsula- } \\
\text { do, granulado }\end{array}$ \\
\hline Porcentaje de ingrediente Activo & $0-100$ \\
\hline Proporción de ingrediente activo & $\begin{array}{l}480 \mathrm{~g} / \mathrm{L}, 482.3 \mathrm{~g} / \mathrm{L}, \\
750 \mathrm{~g} / \mathrm{kg}\end{array}$ \\
\hline Nombre científico de la plaga & $\begin{array}{l}\text { Amaranthus sp., Ipo- } \\
\text { moea purpurea, Capsella } \\
\text { bursapastoris, Datura } \\
\text { stramonium }\end{array}$ \\
\hline Nombre común de la plaga & $\begin{array}{l}\text { Cenicilla, zacate johnson, } \\
\text { quelite, carbón parcial }\end{array}$ \\
\hline Dosis & $\begin{array}{l}0.75-1.5 \mathrm{~L} / \mathrm{ha}, 0.45-0.9 \mathrm{~L} / \mathrm{ha} \text {, } \\
25-30 \mathrm{~kg} / \mathrm{ha}\end{array}$ \\
\hline Límite máximo de residuos & Sin límite \\
\hline Tipo de plaguicida $1 *$ & $\begin{array}{l}\text { Herbicida, insecticida, } \\
\text { fungicida }\end{array}$ \\
\hline Tipo de plaguicida $2 *$ & $\begin{array}{l}\text { Acaricida, desecante, } \\
\text { nematicida, otros }\end{array}$ \\
\hline Uso agrícola & $\begin{array}{l}\text { Aplicación al follaje, } \\
\text { aplicación en control de } \\
\text { malezas, aplicación en } \\
\text { tratamiento de semillas } \\
\text { para siembra, aplicación al } \\
\text { suelo, otros }\end{array}$ \\
\hline
\end{tabular}

Las columnas señaladas con un asterisco $(*)$ se agregaron al archivo original del listado de plaguicidas de uso agrícola (SENASICA 2011)
Con este listado y las precisiones que contiene el catálogo de plaguicidas (COFEPRIS 2015), se generó una base de datos propia para los nueve cultivos mencionados anteriormente, incluyendo los tipos de plaguicidas. Además, se estimó la frecuencia de uso, partiendo de la base de que a mayor oferta de PC corresponde una mayor demanda. Aquellos IA incluidos en un mayor número de $\mathrm{PC}$ se consideraron como los más frecuentemente usados por los productores.

\section{Análisis de ingredientes activos de plaguicidas aplicados por aspersión}

En este análisis se resumieron los resultados en relación con la peligrosidad de los plaguicidas y a sus efectos adversos a la salud, así como a la probabilidad de que residuos de ellos puedan llegar a las localidades cercanas a campos de cultivo (por frecuencias de uso y cantidades aplicadas), su persistencia y características del ingrediente activo que facilitan su dispersión a zonas externas al campo de cultivo.

Se examinó el carácter de peligrosidad del plaguicida de acuerdo con el catálogo 2015 de plaguicidas de la Comisión Federal para la Protección contra Riesgos Sanitarios (COFEPRIS), con el manual de la Organización Mundial de la Salud (OMS) y con la literatura científica consultada, con énfasis en lo que se refiere al riesgo de exposición crónica (pasiva) a los ingredientes activos analizados.

Los plaguicidas que se reportan como peligrosos cumplen con dos o más de estas características: a) se aplican sobre más de $10 \%$ del área total del DDR, b) están clasificados en la bibliografía como peligrosos o medianamente peligrosos, c) son de uso intensivo (por cantidades y frecuencias de aplicación), tienen más productos comerciales por principio activo (>10) y d) son moderadamente persistentes en el ambiente o su degradación produce compuestos de igual y/o mayor peligrosidad.

Esta metodología constituye una guía para abordar la problemática de la contaminación pasiva por plaguicidas dentro del modelo: "plaguicidas-exposiciónefectos adversos a la salud".

\section{RESULTADOS}

En el periodo de 2010 a 2014 se sembraron anualmente un promedio de 45 cultivos, donde 29 de ellos ocupabann menos de $1 \%$ de la superficie agrícola del distrito (Cuadro III), lo cual sugiere una diversificación moderada de la agricultura.

El área de siembra agrícola promedio de 2010 a 2014 fue de 72499 ha. Los cultivos de alfalfa, 
CUADRO II. RESUMEN DE INFORMACIÓN OBTENIDA DE FUENTES BIBLIOGRÁFICAS SOBRE ENFERMEDADES Y PLAGAS PREDOMINANTES EN LOS CULTIVOS DE GARBANZO, VID Y TRIGO EN EL DISTRITO DE DESARROLLO RURAL 144 HERMOSILLO

\begin{tabular}{|c|c|c|c|c|}
\hline \multirow{2}{*}{$\begin{array}{l}\text { Cultivo y } \\
\text { referencias }\end{array}$} & \multirow{2}{*}{$\begin{array}{c}\text { Tipo de enfermedades } \\
\text { y plagas }\end{array}$} & \multicolumn{2}{|c|}{ Enfermedades y plagas } & \multirow{2}{*}{$\begin{array}{l}\text { Aplicación de } \\
\text { plaguicidas }\end{array}$} \\
\hline & & Nombre científico & Nombre común & \\
\hline \multirow{3}{*}{$\begin{array}{l}\text { Garbanzo } \\
\text { (Ortega et al. } \\
2014, \\
\text { INIFAP 2010, } \\
\text { SAGARPA, } \\
2015 b \text { ) }\end{array}$} & Malezas & Convolvulus arvensis L. & Correhuela & Follaje \\
\hline & Insectos y arácnidos & $\begin{array}{c}\text { Spodoptera exigua, Helicoverpa zea } \\
\text { Liriomyza huidobrensis B. } \\
\text { Agrotis ípsilon } \\
\text { Melanagromyza obtusa }\end{array}$ & $\begin{array}{l}\text { Gusano soldado, Gusano } \\
\text { bellotero, Mosquita } \\
\text { minadora } \\
\text { Gusano trozador, Mosquita } \\
\text { de la germinación }\end{array}$ & Follaje \\
\hline & Enfermedades & $\begin{array}{l}\text { Rhizoctonia solani, Macrophomina } \\
\text { phaseolina, Fusarium oxisporum, } \\
\text { Sclerotium rolfsii, Fusarium solani, } \\
\text { Peronospora trifoliorum, Uromyces } \\
\text { ciceris-arietini, Cicer arietinum L. }\end{array}$ & $\begin{array}{c}\text { Pudrición radical, } \\
\text { Pudrición seca } \\
\text { Pudrición radical, Pudrición } \\
\text { Pudrición radical, Mildiú } \\
\text { o cenicilla vellosa, Roya o } \\
\text { chahuixtle, rabia }\end{array}$ & $\begin{array}{l}\text { Follaje y } \\
\text { semillas para } \\
\text { siembra }\end{array}$ \\
\hline \multirow{3}{*}{$\begin{array}{l}\text { Vid } \\
\text { (Fu y Del Real } \\
2009, \\
\text { Vázquez 2011, } \\
\text { SAGARPA, } \\
\text { 2015b) }\end{array}$} & Malezas & $\begin{array}{c}\text { Convolvulus arvensis L. Sorghum } \\
\text { halepense L. Avena fatua, Panicum sp. } \\
\text { Cynodon dactylon L. }\end{array}$ & $\begin{array}{l}\text { Correhuela, zacate johnson } \\
\text { Maleza anual, zacate } \\
\text { bermuda }\end{array}$ & $\begin{array}{l}\text { Control de } \\
\text { malezas }\end{array}$ \\
\hline & Insectos y arácnidos & $\begin{array}{c}\text { Erythroneura variabilis, Harrisinae } \\
\text { brillans, Frankliniella spp, } \\
\text { Planococcus ficus }\end{array}$ & $\begin{array}{l}\text { Chicharrita de la vid, } \\
\text { Gusano presidiario, trips, } \\
\text { piojo harinoso }\end{array}$ & Follaje \\
\hline & Enfermedades & $\begin{array}{c}\text { Uncinula necátor, Phyllosticia } \\
\text { lambruscae } \\
\text { Plasmopara viticola, Phymatotrichum } \\
\text { omnivorum }\end{array}$ & $\begin{array}{l}\text { Cenicilla polvorienta, } \\
\text { pudrición negra, mildiu } \\
\text { velloso, pudrición texana }\end{array}$ & Follaje \\
\hline \multirow{3}{*}{$\begin{array}{l}\text { Trigo } \\
\text { (Grajeda et al. } \\
2013 \text {, } \\
\text { Cortés-Jiménez } \\
\text { et al. 2011, } \\
\text { SAGARPA, } \\
\text { 2015b) }\end{array}$} & Malezas & $\begin{array}{l}\text { Avena fatua, Sorghum halepense, } \\
\text { Panicum sp. Cynodon dactylon, } \\
\text { Convolvulus arvensis }\end{array}$ & $\begin{array}{c}\text { Avena silvestre, zacates, } \\
\text { correhuela }\end{array}$ & $\begin{array}{l}\text { Control de } \\
\text { malezas }\end{array}$ \\
\hline & Insectos y arácnidos & $\begin{array}{c}\text { Macrosiphum avenae, Rhopalosiphum } \\
\text { maidis, Schizaphis graminum, } \\
\text { Mythimna unipuncta }\end{array}$ & Pulgones, gusanos & Follaje \\
\hline & Enfermedades & $\begin{array}{l}\text { Helminthosporium } \mathrm{sp} . \text { Septoria } \mathrm{sp} . \\
\text { Puccinia graminis, Puccinia recóndita } \\
\text { Puccinia triticina, Neovossia indica }\end{array}$ & $\begin{array}{l}\text { Tizón foliar, mancha foliar, } \\
\text { royas, carbón parcial }\end{array}$ & $\begin{array}{l}\text { Follaje y } \\
\text { semillas para } \\
\text { siembra }\end{array}$ \\
\hline
\end{tabular}

CUADRO III. PROMEDIO Y PORCENTAJE DE SUPERFICIE SEMBRADA Y COSECHADA POR AÑO DE LOS CULTIVOS REGISTRADOS, NÚMERO DE CULTIVOS Y NÚMERO DE CULTIVOS CON MÁS DEL 1 \% DE SUPERFICIE TOTAL SEMBRADA DEL DISTRITO DE DESARROLLO RURAL 144 HERMOSILLO, SONORA, MÉXICO

\begin{tabular}{lcccc}
\hline Año & $\begin{array}{c}\text { Superficie } \\
\text { sembrada } \\
\text { (ha) }\end{array}$ & $\begin{array}{c}\text { Superficie } \\
\text { cosechada } \\
\text { (ha) }\end{array}$ & $\begin{array}{c}\text { Número } \\
\text { de } \\
\text { cultivos }\end{array}$ & $\begin{array}{c}\text { Número de } \\
\text { cultivos con } \\
\text { más de 1 \% }\end{array}$ \\
\hline 2010 & 71998 & 71633 & 45 & 15 \\
2011 & 72462 & 70807 & 48 & 16 \\
2012 & 73592 & 71806 & 46 & 16 \\
2013 & 74193 & 71719 & 47 & 16 \\
2014 & 70249 & 67870 & 45 & 16 \\
Promedio & 72499 & 70767 & 45 & 16 \\
\hline
\end{tabular}

Fuente: Datos compilados de las bases de datos de cultivos en Sonora para los años mencionados del Servicio de Información Agroalimentaria y Pesquera (SIAP 2015) 
calabaza, garbanzo, naranja, nogal, sandía, sorgo, trigo y vid ocupaban $80 \%$ de la superficie del DDR (Cuadro IV). De acuerdo con el sistema de clasificación industrial de América del norte (SCIAN), de los cultivos con mayor superficie sembrada en el periodo 2010-2014, están dos cereales (7449 ha), uno de ellos el trigo como predominante, además de seis cultivos forrajeros (12825 ha), en el que destacó la alfalfa verde y el sorgo. En frutales hubo 11 cultivos ( $23081 \mathrm{ha}$ ), entre los que se destacaron la vid, el nogal y la naranja. En hortalizas hubo 23 cultivos (9070 ha) en los que sobresalió la calabaza y la sandía, mientras que en leguminosas hubo dos cultivos (14625 ha), en los que resaltó el garbanzo y en oleaginosas el único sembrado fue el cártamo (3199 ha) (INEGI 2013).

Respecto a los plaguicidas es importante considerar la estacionalidad de los cultivos. En los que fueron estudiados hubo cuatro perennes: alfalfa (3 años), naranja, nogal y vid (6-8 años), el resto de fueron anuales o estacionales. Aunque la producción agrícola cubre prácticamente todo el año, los meses en los que se aplican más plaguicidas por aspersión son de febrero a mayo, principalmente en los cultivos de garbanzo, trigo y vid. De septiembre a noviembre en cultivos como calabaza, nogal, naranja, sandía y sorgo forrajero (Fig. 2). La información relativa a estos cultivos junto con los plaguicidas que se aplican

CUADRO IV. SUPERFICIES SEMBRADAS EN EL PERIODO 20102014 DE LOS OCHO CULTIVOS CON MAYOR SUPERFICIE EN EL DISTRITO DE DESARROLLO RURAL144 HERMOSILLO, SONORA, MÉXICO

\begin{tabular}{lrrrrr}
\hline Cultivo & \multicolumn{5}{c}{ Años } \\
\cline { 2 - 6 } & 2010 & 2011 & 2012 & 2013 & 2014 \\
\hline Trigo & 12881 & 11960 & 11777 & 8502 & 7442 \\
Vid & 10972 & 10912 & 10325 & 11843 & 11843 \\
Garbanzo & 10100 & 8700 & 8969 & 13285 & 13700 \\
Nuez & 6501 & 6793 & 6817 & 7496 & 7496 \\
Alfalfa verde & 4358 & 4358 & 4358 & 4358 & 3608 \\
Calabaza & & 4656 & 5129 & 4755 & 4399 \\
Naranja & 4480 & 3970 & 3564 & & 3037 \\
Sandia & 3554 & & 3500 & 3356 & \\
Sorgo forrajero & 3310 & 3802 & & 3122 & \\
Cártamo & & & & & 3199 \\
Total & 56156 & 55151 & 54439 & 56717 & 54724 \\
\hline
\end{tabular}

Datos compilados de las bases de datos de cultivos en Sonora para los años mencionados del Servicio de Información Agroalimentaria y Pesquera (SIAP 2015)

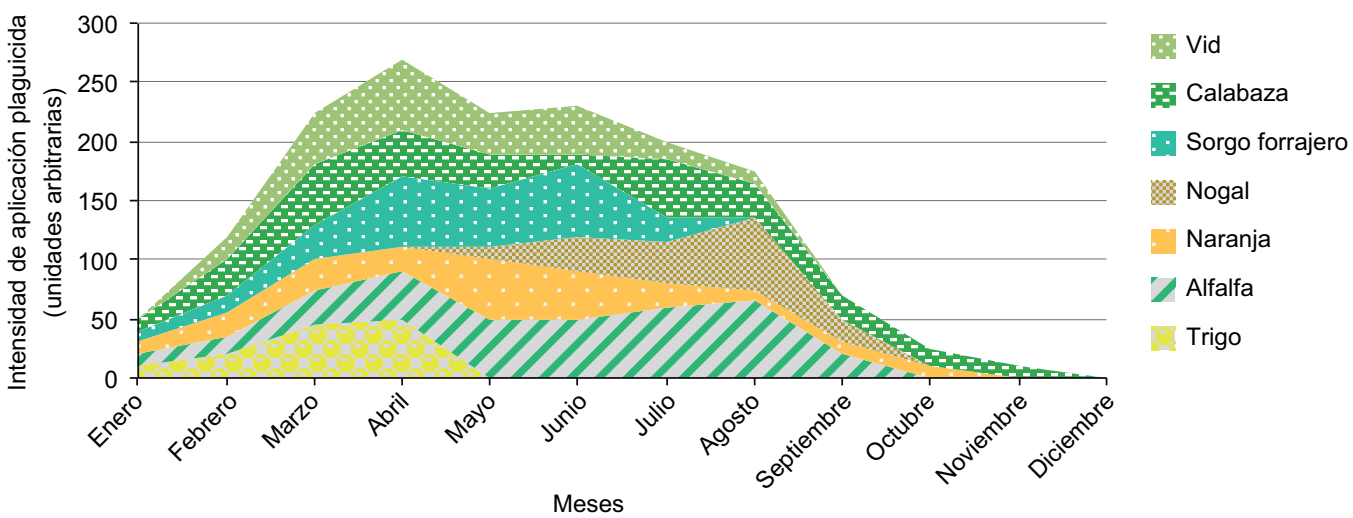

Fig. 2. Estacionalidad de la aplicación de plaguicidas para los cultivos descritos en este estudio en el Distrito de Desarrollo Rural 144 Hermosillo Fuente: SAGARPA (2015b) 
se puede hacer extensiva a otros distritos, ya que a excepción del cártamo, papa y espárrago, la mayor superficie sembrada en Sonora corresponde a los nueve cultivos seleccionados (INEGI 2014).

El listado de plaguicidas de uso agrícola (SENASICA 2011), reporta agroquímicos biológicos y químicos. En el garbanzo se están usando $25 \%$ de plaguicidas biológicos, mientras que en el resto de los cultivos se reporta sólo $5 \%$ de plaguicidas naturales y/o biológicos. En los plaguicidas químicos, los ingredientes activos (IA) que se aplican ya sea por aspersión o directamente a las malezas son 116, con un promedio de 64 productos comerciales (PC) por IA. En este cálculo no se consideran los PC que contienen más de un IA, hay 1104 PC que son mezclas de dos o más IA (276).

Es importante considerar la peligrosidad de un plaguicida en función de la posible dispersión aérea que pueda llevar residuos hasta las localidades rurales, centros escolares u otros sitios habitados. Es por ello que reciben especial atención los que se utilizan en cultivos como nogal y naranjo, dado que se aplican a cierta altura que favorece su dispersión por el aire en función de ciertos parámetros ambientales, lo cual también puede ocurrir con la vid. En cambio, los herbicidas se aplican directamente a las malezas y tienen un menor alcance en las mismas condiciones ambientales (Damalas 2015).

En el cuadro $\mathbf{V}$ se presenta un resumen de los principales ingredientes activos (IA) que se aplican por aspersión en los nueve cultivos estudiados, además incluye la especificación del principio activo, los cultivos donde se aplican, la superficie del DDR que cubren, su dosis promedio y el promedio de PC por IA. De estos resultados se puede estimar que en $65 \%$ del área promedio sembrada en el DDR 144 Hermosillo, se aplican al menos una vez en cada época de siembra 211280 L de glifosato, 70427 L de malatión, 58689 L de diazinón y 46951 L de paratión metílico. Predominan los insecticidas organofosforados (metil

CUADRO V. PLAGUICIDAS APLICADOS POR ASPERSIÓN CON MAYOR FRECUENCIA EN NUEVE CULTIVOS QUE OCUPAN 80 \% DEL ÁREA SEMBRADA EN EL DISTRITO DE DESARROLLO RURAL 144 HERMOSILLO

\begin{tabular}{|c|c|c|c|c|c|c|}
\hline Plaguicidas $^{1}$ & Cultivos & $\begin{array}{c}\text { Área } \\
\text { aplicada }\end{array}$ & $\begin{array}{l}\% \text { Área } \\
\text { de DDR }\end{array}$ & $\begin{array}{l}\text { Promedio } \\
\text { PC por IA }^{2}\end{array}$ & $\begin{array}{c}\text { Dosis } \\
\text { promedio }^{3}\end{array}$ & $\begin{array}{c}\text { Total } \\
\text { aplicado }^{3}\end{array}$ \\
\hline $2,4-\mathrm{D}$ & alfalfa, trigo, vid & 13103 & 18.07 & 43 & 1.25 & 16379 \\
\hline Azinfos metílico & alfalfa, naranjo, nogal, sorgo, trigo, vid & 39417 & 54.37 & 21 & 1.5 & 59126 \\
\hline Benomilo & calabaza, naranjo, sorgo, vid & 22605 & 31.18 & 12 & 0.6 & 13563 \\
\hline Bifentrina & calabaza, naranjo, vid & 19453 & 26.83 & 3 & 0.5 & 9727 \\
\hline Captan & calabaza, sandía, vid & 19003 & 26.21 & 5 & 2.5 & 47508 \\
\hline Clorotalonil & naranjo, sandía & 20637 & 28.47 & 25 & 2 & 41274 \\
\hline Etil clorpirifos & alfalfa, calabaza, garbanzo, sandía, sorgo, trigo & 32150 & 44.35 & 10 & 0.9 & 28935 \\
\hline Deltametrina & calabaza, garbanzo, sandía, sorgo & 21908 & 30.22 & 3 & 0.25 & 5477 \\
\hline Diazinon & $\begin{array}{l}\text { alfalfa, calabaza, naranjo, nogal, sandía, sorgo, } \\
\text { trigo, vid }\end{array}$ & 46951 & 64.76 & 25 & 1.25 & 58689 \\
\hline Dimetoato & alfalfa, naranjo, nogal, sandía, trigo, vid & 39432 & 54.39 & 41 & 0.9 & 35489 \\
\hline Diurón & sorgo, vid & 17788 & 24.54 & 17 & 2.7 & 48028 \\
\hline \multirow{2}{*}{$\begin{array}{l}\text { Endosulfan } \\
\text { Glifosato }\end{array}$} & alfalfa, calabaza, nogal, sandía, sorgo, trigo, vid & 43334 & 59.77 & 28 & 1.75 & 75835 \\
\hline & $\begin{array}{l}\text { alfalfa, calabaza, naranjo, nogal, sandía, sorgo, } \\
\text { trigo, vid }\end{array}$ & 46951 & 64.76 & 65 & 4.5 & 211280 \\
\hline Imidacloprid & alfalfa, calabaza, naranjo, sandía, vid & 26363 & 36.36 & 10 & 0.75 & 19772 \\
\hline Malation & $\begin{array}{l}\text { alfalfa, calabaza, naranjo, nogal, sandía, sorgo, } \\
\text { trigo, vid }\end{array}$ & 46951 & 64.76 & 66 & 1.5 & 70427 \\
\hline Mancozeb & sandía, sorgo, trigo, vid & 27739 & 38.26 & 21 & 2.7 & 74895 \\
\hline Metamidofos & alfalfa, calabaza, sandía & 11742 & 16.2 & 10 & 1.25 & 14678 \\
\hline Metidation & alfalfa, naranjo, nogal, sandía, sorgo & 17997 & 24.82 & 15 & 0.75 & 13498 \\
\hline Metomilo & alfalfa, calabaza, sandía, sorgo, trigo, vid & 36314 & 50.09 & 21 & 0.7 & 25420 \\
\hline Mevinfos & alfalfa, sandía, sorgo & 7360 & 10.15 & 5 & 0.2 & 1472 \\
\hline Paraquat & alfalfa, naranjo, nogal, sandía, sorgo, trigo, vid & 42584 & 58.74 & 50 & 2.25 & 95814 \\
\hline Paratión metílico & $\begin{array}{l}\text { alfalfa, calabaza, naranjo, nogal, sandía, sorgo, } \\
\text { trigo, vid }\end{array}$ & 46951 & 64.76 & 56 & 1 & 46951 \\
\hline Pyraclostrobin & alfalfa, calabaza, garbanzo, sandía, trigo & 21693 & 29.92 & 1 & 0.5 & 10847 \\
\hline
\end{tabular}

${ }^{1}$ Aplicados al follaje o al combate de maleza, ${ }^{2}$ IA con más productos comerciales ofrecidos según el listado de plaguicidas (SENASICA 2011), ${ }^{3} \mathrm{En} \mathrm{L} / \mathrm{ha}$ o en $\mathrm{kg} /$ ha según presentación. $\mathrm{PC}=$ Per cápita, IA = Ingrediente activo

Fuente: Compilado de datos obtenidos de SIAP (2015) y del catálogo de plaguicidas de SENASICA (2011) 
azinfos, etil clorpirifos, diazinon, dimetoato, malatión y paratión metílico), seguido de los herbicidas 2,4-D, endosulfan, glifosato y paraquat, y en menor proporción los fungicidas clorotalonil y mancozeb.

Los plaguicidas más usados por los productores en sus cultivos (IA con mayor cantidad de PC) fueron: malatión en ocho cultivos (66 PC), glifosato en ocho cultivos (65 PC), paratión metílico en ocho cultivos (56 PC), paraquat en siete cultivos (50 PC), el herbicida 2,4-D (43 PC) en tres cultivos y el insecticida dimetoato (41 PC) en seis cultivos.

En el cuadro VI se resume a aquellos plaguicidas que representan un mayor peligro para los habitantes de comunidades rurales del distrito. Dentro de los

CUADRO VI. CARACTERÍSTICAS DE PERSISTENCIA Y PELIGROSIDAD PARA LA SALUD DE INGREDIENTES ACTIVOS DE PLAGUICIDAS AGRÍCOLAS APLICADOS POR ASPERSIÓN EN EL DISTRITO DE DESARROLLO RURAL (DDR) 144 HERMOSILLO

\begin{tabular}{|c|c|c|c|c|c|c|}
\hline \multirow[b]{2}{*}{$\begin{array}{l}\text { Ingrediente } \\
\text { activo }\end{array}$} & \multirow[b]{2}{*}{$\begin{array}{l}\text { Grupo } \\
\text { químico }\end{array}$} & \multirow[b]{2}{*}{$\begin{array}{l}\text { Persistencia en } \\
\text { ambiente }\end{array}$} & \multicolumn{4}{|c|}{ Efectos adversos a la salud (crónicos) reportados por OMS, COFEPRIS, IARC } \\
\hline & & & $\begin{array}{l}\text { Carcinógenos, } \\
\text { mutagénicos, } \\
\text { teratogénicos }\end{array}$ & $\begin{array}{c}\text { Tóxico } \\
\text { reproductivo y del } \\
\text { desarrollo }\end{array}$ & $\begin{array}{l}\text { Inhibidor } \\
\text { colinesterasa } \\
\text { Neurotóxico }\end{array}$ & Otros daños \\
\hline $\begin{array}{l}\text { Clorotalonil } \\
\text { (Fungicida) }\end{array}$ & $\begin{array}{l}\text { Aromático } \\
\text { policlorado }\end{array}$ & $\begin{array}{l}\text { Hasta } 1 \text { año, baja } \\
\text { movilidad }\end{array}$ & Carcinógeno & & & \\
\hline $\begin{array}{l}\text { 2,4-D } \\
\text { (Herbicida) }\end{array}$ & Clorofenoxi & Varios meses & $\begin{array}{c}\text { Probable } \\
\text { carcinógeno }\end{array}$ & & Neurotóxico & \\
\hline $\begin{array}{l}\text { Paratión metílico } \\
\text { (Insecticida) }\end{array}$ & $\begin{array}{l}\text { Organo- } \\
\text { fosforado }\end{array}$ & Días a meses & $\begin{array}{l}\text { Posible } \\
\text { carcinógeno, } \\
\text { Teratogénico }\end{array}$ & $\begin{array}{l}\text { Tóxico del } \\
\text { desarrollo }\end{array}$ & $\begin{array}{c}\text { Inhibidor } \\
\text { colinesterasa } \\
\text { Neurotóxico }\end{array}$ & \\
\hline $\begin{array}{l}\text { Endosulfán } \\
\text { (Insecticida) }\end{array}$ & $\begin{array}{l}\text { Organo- } \\
\text { clorado }\end{array}$ & Muy persistente & $\begin{array}{l}\text { Posibles efectos } \\
\text { teratogénicos }\end{array}$ & & & $\begin{array}{l}\text { Efectos en hígado y } \\
\text { riñón }\end{array}$ \\
\hline $\begin{array}{l}\text { Paraquat } \\
\text { (Herbicida) }\end{array}$ & Bipiridilo & $\begin{array}{c}\text { Altamente } \\
\text { persistente (hasta } 3 \\
\text { años) }\end{array}$ & $\begin{array}{l}\text { Probable } \\
\text { mutagénico } \\
\text { Posible } \\
\text { carcinógeno }\end{array}$ & Reproductivo & & $\begin{array}{l}\text { Hígado, pulmones, } \\
\text { corazón, riñones, } \\
\text { córnea, glándulas } \\
\text { adrenales, piel y } \\
\text { sistema }\end{array}$ \\
\hline $\begin{array}{l}\text { Glifosato } \\
\text { (Herbicida) }\end{array}$ & $\begin{array}{l}\text { Fosforo- } \\
\text { metilglicina }\end{array}$ & $\begin{array}{c}\text { Ligeramente } \\
\text { persistente (14 a } 22 \\
\text { días) }\end{array}$ & $\begin{array}{c}\text { Posible } \\
\text { carcinógeno por } \\
\text { sus aditivos }\end{array}$ & & & $\begin{array}{l}\text { Se han reportado } \\
\text { efectos } \\
\text { hematológicos } \\
\text { Efectos adversos en } \\
\text { ojos e hígado }\end{array}$ \\
\hline $\begin{array}{l}\text { Malatión } \\
\text { (Insecticida) }\end{array}$ & $\begin{array}{l}\text { Organo- } \\
\text { fosforado }\end{array}$ & $\begin{array}{l}\text { Ligeramente } \\
\text { persistente (hasta } \\
\text { una semana). }\end{array}$ & Mutagénico. & & $\begin{array}{c}\text { Inhibidor } \\
\text { colinesterasa }\end{array}$ & \\
\hline Clorpirifos & $\begin{array}{l}\text { Organo- } \\
\text { fosforado }\end{array}$ & $\begin{array}{c}\text { Moderadamente } \\
\text { persistente (un año) }\end{array}$ & & $\begin{array}{l}\text { Efectos } \\
\text { reproductivos en } \\
\text { animales }\end{array}$ & Neurotóxico & $\begin{array}{c}\text { Tóxico para el } \\
\text { sistema respiratorio } \\
\text { y cardiovascular }\end{array}$ \\
\hline $\begin{array}{l}\text { Azinfos metílico } \\
\text { (Insecticida) }\end{array}$ & $\begin{array}{l}\text { Organo- } \\
\text { fosforado }\end{array}$ & $\begin{array}{l}\text { Ligeramente } \\
\text { persistente y con } \\
\text { baja movilidad. }\end{array}$ & & $\begin{array}{l}\text { Posibles efectos } \\
\text { reproductivos en } \\
\text { humanos }\end{array}$ & $\begin{array}{l}\text { Inhibición de } \\
\text { la colinesterasa } \\
\text { eritrocitaria y } \\
\text { plasmática }\end{array}$ & \\
\hline $\begin{array}{l}\text { Mancozeb } \\
\text { (Fungicida) }\end{array}$ & $\begin{array}{l}\text { Ditio- } \\
\text { carbamato }\end{array}$ & $\begin{array}{c}\text { Vida media de } 1 \text { a } \\
7 \text { días }\end{array}$ & $\begin{array}{l}\text { Posible } \\
\text { teratogénico y } \\
\text { carcinógeno }\end{array}$ & & & Daño a la tiroides \\
\hline
\end{tabular}

IARC $=$ International Agency for Research on Cancer, COFEPRIS = Comisión Federal para la Protección contra Riesgos Sanitarios, OMS = Organización Mundial de la Salud

Fuente: De la base de datos de plaguicidas para nueve cultivos del DDR Hermosillo, obtenida del listado de plaguicidas agrícolas de SENASICA (2011), COFEPRIS (2015), Loomis et al. 2015 y literatura citada en este estudio 
cultivos de este estudio los insecticidas empleados con mayor frecuencia fueron malatión, paratión metílico, diazinon, dimetoato, metil azinfos y etil clorpirifos. Estos insecticidas organofosforados son considerados desde ligera a extremadamente peligrosos, se aplican en el $90 \%$ de los cultivos y su toxicidad puede afectar la salud humana y deteriorar el ambiente. El cuadro VI resume los efectos de los plaguicidas más utilizados en DDR 144 Hermosillo; el paratión metílico está listado por la OMS como extremadamente peligroso (Ia), mientras que el metil azinfos es altamente peligroso (Ib). Clorpirifos, dimetoato y diazinon son medianamente peligrosos, mientras que malatión es inhibidor de la colinesterasa y tiene efectos mutagénicos. Entre los herbicidas destaca el glifosato (Cuadro VI), considerado como probable carcinógeno según la Agencia Internacional de Investigación en Cáncer (IARC); el paraquat que actualmente está restringido por su toxicidad y persistencia, y el 2,4-D como posible carcinógeno y neurotóxico (COFEPRIS 2015, Loomis et al. 2015).

De los fungicidas el clorotalonil es cancerígeno con persistencia en el ambiente, mientras que el mancozeb se considera un posible teratogénico y disrruptor endócrino (tiroides), aunque su persistencia en el medio es muy baja (WHO 2010, COFEPRIS 2015).

\section{DISCUSIÓN}

El área agrícola del DDR 144 Hermosillo está formada por predios agrícolas de agricultura comercial y de riego, donde $80 \%$ de la misma, la ocupan nueve cultivos que usan 24 IA de plaguicidas aplicados por aspersión durante la temporada de desarrollo del cultivo. Las cantidades de éstos varían con el plaguicida y van desde 16 a $211 \mathrm{t}$ por temporada. Los insecticidas organofosforados, el endosulfán, herbicidas (2,4-D, paraquat y glifosato) y fungicidas (clorotalonil y mancozeb) son los que predominan, es decir, que son aplicados con mayor frecuencia. La mayoría de ellos tienen efectos nocivos para la salud. Cabe señalar que varios de estos contaminantes han sido prohibidos o restringidos en ciertos países por la alta incidencia de efectos adversos graves e irreversibles ocasionados en la salud humana y en el ambiente. Sin embargo, en México se continúan aplicando como lo demuestran los estudios en el noroeste de México (González et al. 2010, Hernández y Hansen 2011, Leal et al. 2014, Leyva et al. 2014), incluyendo este estudio.
Al no contar con estadísticas oficiales del uso y consumo de plaguicidas en el área agrícola, la información disponible es muy general y no está diferenciada para cultivos anuales y perennes, así como para cultivos extensivos e intensivos, árboles frutales y otras categorías en las cuales puede variar la forma en que se aplican y su efecto en el ambiente (Albert 2015). Las estimaciones más recientes son de los trabajos de González et al. (2010) y de Leyva et al. (2014). El estudio de González et al. (2010) se realizó en 19 municipios y 90 establecimientos de venta de agroquímicos en el estado de Nayarit, México. Se obtuvo información sobre la frecuencia de venta, sus marcas comerciales, así como las épocas y plaguicidas con mayor venta. Mientras que el trabajo de Leyva et al. (2014), se realizó en cuatro distritos de riego del Valle de Culiacán, Sinaloa, México, mediante compilación de datos sobre plaguicidas del programa "campo limpio" promovido por el Servicio Nacional de Sanidad, Inocuidad y Calidad Agroalimentaria (SENASICA) y de bitácoras de empresas agrícolas de la zona.

En lo que respecta a las cantidades de plaguicidas aplicados por aspersión para los nueve cultivos (Cuadro V, éstas coinciden en la aplicación de organofosforados con las estimaciones realizadas por González et al. (2010), así como por las de Leyva et al. (2014). Mientras que en los estados de Nayarit y Sinaloa los fungicidas fueron predominantes seguidos de herbicidas e insecticidas. En el presente estudio, al considerarse solamente los plaguicidas aplicados por aspersión, hay mayor predominancia de insecticidas y herbicidas, ya que muchos de los fungicidas se aplican al suelo o a la semilla para siembra. Asimismo, en ambos trabajos se mencionan los insecticidas organofosforados como los predominantes, similares a las obtenidas en este trabajo. A pesar de que México ha incorporado recientemente en su legislación federal varias normas nacionales e internacionales referentes a los plaguicidas (Albert 2015), éstas no han llegado a plasmarse o incorporarse a la legislación estatal y municipal. No existen los registros sobre el uso de plaguicidas a nivel de predios agrícolas, así como tampoco del cumplimiento de las medidas preventivas establecidas en las normas federales.

El aumento constante del uso de plaguicidas en la agricultura es un problema mundial, sobre todo en la producción de alimentos (Albert 2015). Los aspectos que se consideran para la reducción del uso de agroquímicos son los de la preservación del ambiente junto con la reducción de daños que pudiera causar el cambio climático (Chakraborty y 
Newton 2011). Además de la reducción del riesgo de contaminación por plaguicidas en aquellas poblaciones potencialmente expuestas (Eyhorn et al., 2015). Una forma de estimar la dispersión de los plaguicidas es la implementación y validación de modelos empíricos de su dispersión, que estimen la distancia hasta la que pueden llegar sus residuos, así como su concentración (Alavanja et al. 2002, Donatelli et al. 2009, Nuyttens et al. 2010, Fritz et al. 2011, Butler-Ellis et al. 2016). Estos modelos podrían ser realizados por los propios agricultores tomando en cuenta el plaguicida que se aplica por aspersión, el cultivo, las características de los equipos aspersores y datos climatológicos relevantes para la dispersión del plaguicida tales como la temperatura, la humedad y los vientos, entre otros. Estos últimos pueden obtenerse de estaciones agro climatológicas propias o del sistema de la Red de Estaciones Meteorológicas Automatizadas de Sonora (REMAS) (SIAFESON 2016), el cual reporta datos climáticos para la agricultura en tiempo real. Con estos modelos se podrían determinar zonas "amortiguadoras", el efecto de barreras arboladas y las distancias mínimas entre campos y localidades que prevengan una mayor contaminación en zonas habitadas.

Se han propuesto acciones de sustentabilidad ambiental en la agricultura, tales como el empleo del control integrado de plagas, prácticas culturales de control, uso de microorganismos e insectos benéficos y reducción de las aplicaciones de plaguicidas, las cuales beneficiarán en el largo plazo la sustentabilidad ambiental de los cultivos, aunque en el corto plazo puedan significar cierta reducción en su rentabilidad (Mostafalou y Abdollahi 2012, Riojas et al. 2013, Márquez et al. 2014, Eyhorn et al. 2015). En lo que se refiere a los cultivos, Lechenet et al. (2014), señalan que entre las alternativas disponibles con mínimo efecto sobre la rentabilidad de los cultivos pueden ser el uso de sistemas agrícolas integrados que promueven la diversificación y rotación de cultivos, así como la combinación de una amplia gama de técnicas disponibles que contribuyen al manejo de las plagas. En su trabajo los autores comprueban que el manejo integrado de plagas no afecta la rentabilidad de los cultivos ni tiene una mayor carga de mano de obra si se mantiene un sistema de rotación de cultivos.

Existen propuestas de medidas punitivas para los dueños de predios agrícolas, tales como la aplicación de gravámenes tributarios a los plaguicidas (Cartujano et al. 2015) o de responsabilidad ambiental en el caso de comprobarse efectos en la salud de poblaciones cercanas a campos agrícolas (Ortiz et al. 2013).
La literatura actual sobre el riesgo de los plaguicidas agrícolas señala que la evaluación de su riesgo debe asociar las vías de exposición con los resultados de estudios de biomonitoreo epidemiológico de las poblaciones humanas expuestas (Ross et al. 2015). Ya existe evidencia empírica de la presencia de residuos de plaguicidas (o de biomarcadores de daño por plaguicidas) en las poblaciones agrícolas de Sonora. Sin embargo, aún falta entender la cuota de riesgo que representa cada ruta de exposición, ya que ésta es a través de múltiples vías. Las que resaltan son: el trabajo o la aplicación de plaguicidas en la agricultura, la dispersión de plaguicidas de campos agrícolas cercanos, el arrastre de plaguicidas en ropas y equipo de trabajadores agrícolas que habitan en dichas localidades, así como la aplicación residencial de plaguicidas (Deziel et al. 2013, Arcury et al. 2014).

Los resultados de este estudio pueden servir para seleccionar los plaguicidas a estudiar o escoger las épocas del año en que éstos pueden llegar con mayor intensidad a las localidades, centros escolares y/o sitios públicos en los alrededores de campos de cultivo. Además, pueden servir como antecedente para estudios de biomonitoreo de plaguicidas de corte longitudinal integrado para delimitar las vías de exposición más relevantes en grupos de población humana vulnerable a los efectos adversos de los plaguicidas (Mitchell et al. 2013). Por otro lado, los recientes avances en las capacidades de recolección de datos sobre exposición a plaguicidas y en metodologías predictivas, permiten el desarrollo de planes sistemáticos. Lo anterior para asignar prioridades en el análisis de químicos de fuentes difusas o indirectas, asociados con la presencia de plaguicidas en biomonitoreos ambientales o humanos específicos. A estos análisis se le adicionan los modos de entrada al ambiente y/o al organismo humano (Mitchell et al. 2013, Ramírez et al. 2015, VoPham et al. 2015).

Algunas de las limitaciones de este trabajo consisten en que no se contemplan ciertos cultivos (cártamo, papa y espárrago, entre otros) por su baja superficie de siembra en el DDR 144 Hermosillo, los cuales podrían ser relevantes en otros distritos de Sonora.

Quizá la limitación más importante es que se usan datos que pudieran estar actualmente obsoletos (2010-2014) al no contar con información actualizada sobre el uso de plaguicidas en el campo. Al respecto, la última guía técnica para Sonora (SAGARPA 2015b) hace énfasis en el uso de métodos culturales para la erradicación de malezas, así como de varios bioplaguicidas o extractos de plantas que podrían aplicarse para el combate integrado de plagas. 


\section{CONCLUSIONES}

Los insecticidas organofosforados, herbicidas $\mathrm{y}$ algunos fungicidas son los que se aplicaron en mayor cantidad y con mayor frecuencia. La mayoría de ellos son de mediana a alta persistencia, están clasificados como mediana a extremadamente dañinos para la salud y están restringidos y/o prohibidos en otros países. La información obtenida puede aplicarse a otros distritos rurales de la región con una mayor superficie sembrada y con los mismos cultivos citados. Este estudio no mide la exposición a plaguicidas, por lo que sus resultados no predicen posibles impactos en la salud. Se requiere información adicional del metabolismo, transporte y vías de exposición de los compuestos estudiados. Sin embargo, la metodología y los resultados pueden ayudar en la planeación de estudios de riesgo como biomonitoreo de plaguicidas en poblaciones humanas rurales y en el ambiente, así como en el diseño de programas preventivos de salud.

\section{AGRADECIMIENTOS}

Se agradece a la Dirección de Investigación y Posgrado y a la Dirección de la División de Ciencias Biológicas y de la Salud de la Universidad de Sonora por su apoyo en el proyecto "Diseño e implementación de modelos integrales de riesgo de exposición a plaguicidas en Sonora", del cual se deriva este artículo.

\section{REFERENCIAS}

Alavanja-Michael C.R., Rowland-Andrew S., Mage D., Hoar-Zahm S., Rothman N., Lubin-Jay H., Dosemeci M., Hoppin-Jane A., Sandler-Dale P. y Blair A. (2002). A quantitative approach for estimating exposure to pesticides in the agricultural health study. Ann. Occup. Hyg. 46 (2), 245-260.

DOI: 10.1093/annhyg/mef011

Albert L.A. (2015). Panorama de los plaguicidas en México [en línea]. http://alef.mx/el-jarocho-cuantico49-los-plaguicidas-en-mexico/ 17/04/2017.

Aldana-Madrid M.L., Valdez-Hurtado S., Vargas-Valdez N.D., Salazar-López N.J. Silveira-Gramont M.I., Loarca-Piña F.G., Rodríguez-Olivarría G., WongCorral F.J., Borboa-Flores J. y Burgos-Hernández A. (2008a). Insecticide residues in stored grains in Sonora, Mexico: Quantification and toxicity testing. Bull. Environ. Contam. Toxicol. 80 (2), 93-96.

DOI: $10.1007 / \mathrm{s} 00128-007-9302-8$
Aldana-Madrid M.L., García-Moraga M.C., RodríguezOlibarria G., Silveira-Gramont M.I. y ValenzuelaQuintanar A.I. (2008b). Determinación de insecticidas organofosforados en nopal fresco y deshidratado, mediante extracción en fase-sólida dispersiva. Rev. Fitotec. Mex. 31 (2), 133-139.

Aldana-Madrid M.L., Valenzuela-Quintanar A.I., SilveiraGramont M.I., Rodríguez-Olibarría G., Grajeda-Cota P., Zuno-Floriano F.G. y Miller M. (2011). Residual pyrethroids in fresh horticultural products in Sonora, Mexico. Bull. Environ. Contam. Toxicol. 87 (4), 44364439. DOI: $10.1007 / \mathrm{s} 00128-011-0391-\mathrm{z}$

Aldana-Madrid M.L., Silveira-Gramont M.I., Zuno-Floriano F.G. y Rodríguez-Olibarría G. (2013). Insecticide residuality of Mexican populations occupationally exposed. En: Insecticides: development of safer and more effective technologies (S. Trdan, Ed.). InTech, Rijeka, Croacia, pp. 311-329.

Arcury T.A., Lu C., Chen H. y Quandt S.A. (2014). Pesticides present in migrant farmworker housing in North Carolina. Am. J. Ind. Med. 57 (3), 312-322. DOI: 10.1002/ajim.22232

Butler-Dawson J., Galvin K., Thorne P.S. y Rohlman D.S. (2016). Organophosphorus pesticide exposure and neurobehavioral performance in Latino children living in an orchard community. NeuroToxicology 53, 165-172. DOI: 10.1016/j.neuro.2016.01.009

Butler-Ellis M.C., van de Zande J.C., van den Berg F., Kennedy M.C., O'Sullivan C.M., Jacobs C.M., Fragkoulis G., Spanoghe P., Gerritsen-Ebben R., Frewer L.J. y Charistou A. (2016). The BROWSE: model for predicting exposures of residents and bystanders to agricultural use of plant protection products: an overview. Biosystems Eng. 154, 92-104. DOI: 10.1016/j.biosystemseng.2016.08.017

Cantu-Soto E.U., Meza-Montenegro M.M., ValenzuelaQuintanar A.I., Felix-Fuentes A., Grajeda-Cota P., Balderas-Cortes J.J., Osorio-Rosas C.L., Acuña-García G. y Aguilar-Apodaca M.G. (2011). Residues of organochlorine pesticides in soils from the southern Sonora, Mexico. Bull. Environ. Contam. Toxicol. 87 (5), 556-560. DOI: 10.1007/s00128-011-0353-5

Cartujano-Escobar S., Rosas-Echeverría M.V., ÁlvarezViolante C., Pérez-Ramírez S.V., Ponce- Lázaro P., López-Tarángo R. y Platas-Neri D.A. (2015). Alcances de las medidas tributarias en la lucha contra la contaminación en México. Memorias. Global Conference on Business and Finance. San José, Costa Rica 10 (2), 765-772. 26 al 29 de mayo, 2015.

Cejudo A.L., Meza M.M., Balderas J.J., Mondaca I., Rodríguez R., Renteria A.M. y Félix A. (2012). Exposición a plaguicidas organoclorados en niños indígenas de Potam, Sonora, México. Ra Ximhai 8 (2), 121-127. 
Chakraborty S. y Newton A.C.(2011). Climate change, plant diseases and food security: an overview. Plant Pathol. 60, 2-14. DOI: 10.1111/j.1365-3059.2010.02411.x

COFEPRIS (2015). Catálogo de plaguicidas. Comisión Federal para la Protección contra Riesgos Sanitarios. Secretaría de Salud Pública [en línea].

http://www.cofepris.gob.mx/AZ/Paginas/Plaguicidas $\% 20 y \% 20$ Fertilizantes/CatalogoPlaguicidas.aspx 17/04/2017.

Cortés-Jiménez J.M., Fuentes-Dávila G., Ortiz Enríquez J.E., Tamayo-Esquer L.M., Cortez-Mondaca E., Ortiz-Ávalos A.A., Félix-Valencia P. y Armenta-Cárdenas I. (2011). Agronomía del trigo en el sur de Sonora. Libro técnico No. 6, Instituto Nacional de Investigaciones Forestales Agrícolas y Pecuarias. Centro de Investigación Regional del Noroeste, Campo Experimental Norman E. Borlaug. Ciudad Obregón, Sonora, México, 237 pp.

Damalas C.A. (2015). Pesticide drift: seeking reliable environmental indicators of exposure assessment. En: Environmental indicators (R.H. Armon y O. Hänninen, Eds.). Springer, Dordrecht, Holanda, pp. 251-261.

Donatelli M., Bellocchi G., Habyarimana E., Confalonieri R. y Micale F. (2009). An extensible model library for generating wind speed data. Comput. Electron. Agric. 69, 165-170. DOI: 10.1016/j.compag.2009.07.022

Deziel N.C., Ward M.H., Bell E.M., Whitehead T.P., Gunier R.B., Friesen M.C. y Nuckols J.R. (2013). Temporal variability of pesticide concentrations in homes and implications for attenuation bias in epidemiologic studies. Environ. Health Perspect. 121 (5), 565-571. DOI: 10.1289/ehp.1205811

Eyhorn F., Roner T. y Specking H. (2015). Reducing pesticide use and risks - what action is needed? Briefing paper. Documento informativo. Stiftung Mercator Schweiz [en linea]. https://assets.helvetas.org/downloads/ briefing_paper_pesticide_reduction_including_conclusions.pdf 17/04/2017.

Fenske R.A., Lu C., Negrete M. y Galvin K. (2013). Breaking the take home pesticide exposure pathway for agricultural families: workplace predictors of residential contamination. Am. J Ind. Med. 56 (9), 1063-1071. DOI: 10.1002/ajim.22225

Fritz B.K., Hoffmann W.C., Bagley W.E. y Hewitt A. (2011). Field scale evaluation of spray drift reduction technologies from ground and aerial application systems. Journal of ASTM International 8 (5), 1-11. DOI: $10.1520 / J A I 103457$

Fu-Castillo A.A. y Del Real-Valdez A.A. (2009). Guía para el control de piojo harinoso de la vid. Instituto Nacional de Investigaciones Forestales, Agrícolas y Pecuarias, Centro de Investigación Regional del Noroeste, Campo Experimental Costa de Hermosillo, Sonora, México, 32 pp.
González-Arias C.A., Robledo-Marenco M.L., MedinaDíaz I.M., Velázquez-Fernández J.B., Girón-Pérez M.I., Quintanilla-Vega B., Ostrosky-Wegman P., PérezHerrera N.E. y Rojas-García A.E. (2010). Patrón de uso y venta de plaguicidas en Nayarit, México. Rev. Int. Contam. Ambie. 26 (3), 221-228.

Grajeda-Grajeda J., Ruiz-Corral J., Félix V.P., Jiménez G.E. y Jiménez-Lagunes A. (2013). Enfermedades importantes de algunos cultivos en México. Instituto Nacional de Investigaciones Forestales, Agrícolas y Pecuarias (INIFAP), Centro de Investigación Regional del Noroeste (CIRNO). Campo Experimental Costa de Hermosillo, Sonora, México, 172 pp.

Harsimran K.G. y Harsh G. (2014). Pesticides: environmental impacts and management strategies. En: Pesticides-toxic aspects (M.L. Larramendy y S. Soloneski, Eds.). Intech, Rijeka, Croacia, pp. 187-230. DOI: $10.5772 / 57399$

Hernández-Antonio A. y Hansen A.M. (2011). Uso de plaguicidas en dos zonas agrícolas de México y evaluación de la contaminación de agua y sedimentos. Rev. Int. Contam. Ambie. 27 (2), 115-127.

Hewitt A.J. (2002). The practical use of Agdrift ${ }^{\circledR}$ and other drift exposure models for aerial, ground and orchard spray applications. Australas. J. Ecotoxicol. 8 (2), 7-19.

Huen K., Bradman A., Harley K., Yousefi P., Boyd B.D., Eskenazi B. y Holland N. (2012). Organophosphate pesticide levels in blood and urine of women and newborns living in an agricultural community. Environ. Res. 117, 8-16.

DOI: $10.1016 /$ j.envres.2012.05.005

INEGI (2010). Censo de población y vivienda 2010. Principales resultados por localidad. Instituto Nacional de Estadística, Geografía e Informática [en línea]. http:// www.inegi.org.mx/sistemas/consulta_resultados/ iter2010.aspx? $\mathrm{c}=27329 \& \mathrm{~s}=$ est 22/05/2017.

INEGI (2013). Catálogo del sistema de clasificación industrial de América del norte (SCIAN). Instituto Nacional de Estadística, Geografía e Informática [en línea] http://www3.inegi.org.mx/sistemas/SCIAN/ scian.aspx 17/04/2017.

INEGI (2014). Anuario estadístico del estado de Sonora. Instituto Nacional de Estadística, Geografía e Informática [en línea]. http://www.datatur.sectur.gob.mx/ITxEF_ Docs/SON_ANUARIO_PDF15.pdf 17/04/2017.

INEGI (2016). El sector alimentario en México, 2014. Actualizaciones. Encuesta mensual de la industria manufacturera. Instituto Nacional de Estadística, Geografía e Informática [en línea]. http://www3.inegi.org.mx/sistemas/sisept/default.aspx?t=mamb91\&s $=$ est $\& c=21546$ 24/02/2017.

INIFAP (2010). Guía técnica para el área de influencia del campo experimental costa de Hermosillo. Instituto 
Nacional de Investigaciones Forestales, Agrícolas y Pecuarias. Hermosillo, Sonora, México, 218 pp.

Koureas M., Tsakalof A., Tsatsakis A. y Hadjichristodoulou C. (2012). Systematic review of biomonitoring studies to determine the association between exposure to organophosphorus and pyrethroid insecticides and human health outcomes. Toxicol. Lett. 210 (2), 155168. DOI: 10.1016/j.toxlet.2011.10.007

Leal S.S., Valenzuela Q.A., Gutiérrez C.L., Bermúdez A.M., García H.J., Aldana M.M. Grajeda C.P., Silveira G.M., Meza M.M., Palma D.S., Leyva G.G., Camarena G.B. y Valenzuela N.C. (2014). Residuos de plaguicidas organoclorados en suelos agrícolas. Terra Latinoam. 32 (1), 1-11.

Lechenet M., Bretagnolle V., Bockstaller Ch., Boissinot F., Petit M-S., Petit M.S. y Munier-Jolain N.M. (2014). Reconciling pesticide reduction with economic and environmental sustainability in arable farming. PLoS ONE 9 (6), 1-10. DOI: 10.1371/journal.pone.0097922

Leyva-Morales J.B., García-de la Parra L.M., BastidasBastidas P.J., Astorga-Rodríguez J.E., Bejarano-Trujillo J., Cruz-Hernández A., Martínez-Rodríguez I.E. y Betancourt-Lozano M. (2014). Uso de plaguicidas en un valle agrícola tecnificado en el noroeste de México. Rev. Int. Contam. Ambie. 30 (3), 247-261.

Loomis D., Guyton K., Grosse Y., El Ghissassi F., Bouvard V., Benbrahim-Tallaa L., Guha N., Mattock H. y Straif K. (2015). Carcinogenicity of lindane, DDT, and 2,4-dichlorophenoxyacetic acid. Lancet Oncol. 16 (8), 891-892.

DOI: $10.1016 / \mathrm{S} 1470-2045(15) 00081-9$

Márquez S.R., Almaguer G. y Schwentesius R. y Ayala A.V. (2014). Trigo en Sonora y su contexto nacional e internacional. Colección: Situación, retos y tendencias para el desarrollo rural sustentable. Centro de Estudios para el Desarrollo Rural Sustentable y la Soberanía Alimentaria. México, D.F., México, 210 pp.

Meza-Montenegro M.M., Gandolfi A.J., Santana-Alcántar M.E, Klimecki W.T., Aguilar-Apodaca M.G, Del RíoSalas R, De la O-Villanueva M., Gómez-Álvarez A., Mendivil-Quijada H., Valencia M. y Meza-Figueroa D. (2012). Metals in residential soils and cumulative risk assessment in Yaqui and Mayo agricultural valleys, northern Mexico. Sci. Total Environ. 433, 472-481. DOI: $10.1016 /$ j.scitotenv.2012.06.083

Meza-Montenegro M.M., Valenzuela-Quintanar A.I., Balderas-Cortés J.J., Yáñez-Estrada L., GutiérrezCoronado M.L., Cuevas-Robles A. y Gandolfi A.J. (2013). Exposure assessment of organochlorine pesticides, arsenic, and lead in children from the major agricultural areas in Sonora, Mexico. Arch. Environ. Contam. Toxicol. 64 (3), 519-527.

DOI: $10.1007 / \mathrm{s} 00244-012-9846-4$
Mitchell J., Arnot J.A., Jolliet O., Georgopoulos P.G., Isukapalli S., Dasgupta S., Pandian M., Wambaugh J., Egeghy P., Cohen Hubal E.A. y Vallero D.A. (2013). Comparison of modeling approaches to prioritize chemicals based on estimates of exposure and exposure potential. Sci. Total Environ. 458-460, 555-567.

DOI: 10.1016/j.scitotenv.2013.04.051

Moreno-Villa E.D., Silveira-Gramont M.I., RodríguezOlibarría G., Quintanar-Valenzuela A.I., Meza-Montenegro M. y Aldana-Madrid M.L. (2012). Análisis de piretroides en suelo y agua de zonas agrícolas y urbanas de los valles del Yaqui y Mayo. Rev. Int. Contam. Ambie. 28 (4), 303-310.

Mostafalou, S. y Abdollahi, M. (2012). Concerns of environmental persistence of pesticides and human chronic diseases. Clin. Exp. Pharmacol. S5, e002.

DOI: $10.4172 / 2161-1459 . S 5-\mathrm{e} 002$

Nuyttens D., De Schampheleire M., Brusselman E., Dekeyser D. y Verboven P. (2010). Drift from field crop sprayers using an integrated approach: results of a 5 year study. Memorias. 2010 ASABE Annual International Meeting. Pittsburgh, Pensilvania, EUA. 20 al 23 de junio, 2010. Pp. 1-6.

Oerke E.C. (2006). Crop losses to pests. J. Agric. Sci. 144 (1), 31-43. DOI: $10.1017 / \mathrm{S} 0021859605005708$

Ochoa-Nogales B., Camarena-Gómez B.O., GutiérrezCoronado M.L., Valenzuela-Quintanar A. I. y AldanaMadrid M.L. (2012). Situación del riesgo por la presencia de COP: evidencias del problema y escenarios de solución. Estudios Sociales 20 (2), 233-251.

Ortega P.F., Fierros G.A., Valenzuela V., Acosta J., Padilla I., Gutiérrez E., Rodríguez F., Velarde S. y Aguilar B.Z. (2014). Generación de nuevas variedades de garbanzo con calidad de exportación y resistentes a las enfermedades de la raíz. En: Avances de proyectos financiados por Fundación Produce Sonora A.C. Ciclo 2013-2014. (L.A. Maldonado, G. Martínez y R.L. Grijalva, Eds.). Instituto Nacional de Investigaciones Forestales Agrícolas y Pecuarias, Centro de Investigación Regional del Noroeste, Campo Experimental Costa de Hermosillo, Hermosillo, Sonora, México, pp. 58-67.

Ortíz I., Avila-Chávez M. y Torres L.G. (2013). Revisión. Plaguicidas en México: usos, riesgos y marco regulatorio. Journal RELBAA 4 (1), 26-46.

Quandt S.A., Hernández-Valero M.A., Grzywacz J.G., Hovey J.D., Gonzáles M. y Arcury T.A. (2006). Workplace, household, and personal predictors of pesticide exposure for farmworkers. Environ. Health Perspect. 114 (6), 943-953. DOI: 10.1289/ehp.8529

Ramírez-Santana M., Zúñiga L, Corral S., Sandoval R., Scheepers P.T.J., Van der Velden K., Roeleveld N. y Pancetti F. (2015). Assessing biomarkers and neuro- 
psychological outcomes in rural populations exposed to organophosphate pesticides in Chile-study design and protocol. BMC Public Health 15 (116), 1-9.

DOI: $10.1186 / \mathrm{s} 12889-015-1463-5$

Remoundou K., Brennan M., Hart A. y Frewer L.J. (2014). Pesticide risk perceptions, knowledge, and attitudes of operators, workers, and residents: A review of the literature. Hum. Ecol. Risk Assess. 20 (4), 1113-1138. DOI: $10.1080 / 10807039.2013 .799405$

Riojas-Rodríguez H., Schilmann A., López-Carrillo L. y Finkelman J. (2013). La salud ambiental en México: situación actual y perspectivas futuras. Salud Pública Mex. 55 (6), 638-649.

Ross J., Driver J.H., Lunchick C. y O’Mahony C. (2015). Models for estimating human exposure to pesticides. Outlooks on Pest Management 26 (1), 33-37.

DOI: 10.1564/v26_feb_09

SAGARPA (2015a). III Informe de labores 20142015. Secretaría de Agricultura, Ganadería, Desarrollo Rural, Pesca y Alimentación [en línea]. http:// www.sagarpa.gob.mx/Transparencia/POT_2015/ FRACCION_X/3er_Informe_de_Labores_Sagarpa_2015.pdf 17/04/2017.

SAGARPA (2015b). Agenda técnica agrícola Sonora. 2a ed. Secretaría de Agricultura, Ganadería, Desarrollo Rural, Pesca y Alimentación [en línea]. http://www. inifap.gob.mx/Documents/agendas_tecnologicas/26_ Sonora_2015_SIN.pdf 17/04/2017.

SEDESOL (2015). Catálogo de localidades de Sonora. Unidad de microrregiones. Dirección General Adjunta de Planeación Micro regional. Secretaría de Desarrollo Social [en línea]. http://www.microrregiones.gob.mx/ catloc/Default.aspx?tipo $=$ clave $\&$ campo $=$ mun \&val or $=26$ 17/04/2017.

SENASICA (2011). Listado de plaguicidas de uso agrícola. Servicio Nacional de Sanidad, Inocuidad y Calidad Agroalimentaria. Secretaría de Agricultura, Ganadería, Desarrollo Rural, Pesca y Alimentación [en línea]. http/ www.senasica.gob.mx 17/04/2017.

SIAFESON (2016). Red de estaciones meteorológicas automáticas de Sonora (REMAS). Sistema de alertas fitosanitarias del estado de Sonora - Comité estatal de sanidad vegetal (CESAVE), 2014-2017 [en línea]. http://www.siafeson.com/remas/index.php/ultimosdatos $17 / 04 / 2017$.

Silveira-Gramont M.I., Amarillas V.T., Rodríguez-Olibarría G., Aldana-Madrid, M.L. y Zuno-Floriano F.G. (2011). Valoración del riesgo de exposición a insecticidas organofosforados en adultos del sexo masculino en Sonora, México. Ciencia@UAQ 4 (2), 70-81.
Silveira M.I., Aldana L., Valenzuela A.I., Ochoa C.B., Jasa-Silveira G. y Camarena B. (2016). Necesidades educacionales sobre riesgo de plaguicidas en el contexto socio-ambiental de las comunidades agrícolas de Sonora. Nova Scientia 8 (16), 371-401.

SIAP (2015). Producción anual por estado. Sistema de Información Agropecuaria y Pesquera [en línea]. http:// www.siap.gob.mx/cierre-de-la-produccion-agricolapor-estado/ 17/04/2017.

Teedon P., Galea K.S., MacCalman L., Jones K., Cocker J., Cherrie J.W. y van Tongeren M. (2015). Engaging with community researchers for exposure science: lessons learned from a pesticide biomonitoring study. PLoS ONE 10 (8), 1-17. DOI: 10.1371/journal.pone.0136347

USEPA (1997). Annual spray drift review. Office of Pesticide Programs, Environmental Fate and Effects Division, United States Environmental Protection Agency [en línea]. http://www.epa.gov/scipoly/sap/ meetings/1997/december/spraydrift.htm 17/04/2017.

Vázquez-Villanueva N. (2011). Modelo de la agricultura moderna en México en el siglo XXI. Asociación agrícola de productores de uva de mesa, Fundación Produce, A.C. Instituto Interamericano de Cooperación para la Agricultura y la Coordinadora Nacional de las Fundaciones Produce. Hermosillo, Sonora, México, $92 \mathrm{pp}$.

VoPham T., Wilson J.P., Ruddell D., Rashed T., Brooks M.M., Yuan J-M., Talbott E.O., Chang C-C. H. y Weissfeld J.L. (2015). Linking pesticides and human health: A geographic information system (GIS) and Landsat remote sensing method to estimate agricultural pesticide exposure. Appl. Geogr. 62, 171-181.

DOI: 10.1016/j.apgeog.2015.04.009

Ward M.H., Lubin J., Giglierano J., Colt J.S., Wolter C., Bekiroglu N., Camann D., Hartge P. y Nuckols J.R. (2006). Proximity to crops and residential exposure to agricultural herbicides in Iowa. Environ. Health Perspec. 114 (6), 893-897. DOI: 10.1289/ehp. 8770

Wofford P., Segawa R., Schreider J., Federighi V., Neal R. y Brattesani M. (2014). Community air monitoring for pesticides. Part 3: Using health-based screening levels to evaluate results collected for a year. Environ. Monit. Assess. 186 (3), 1355-1370.

DOI: $10.1007 / \mathrm{s} 10661-013-3394-x$

WHO (2010). The WHO recommended classification of pesticides by hazard and guidelines to classification 2009, World Health Organization. Ginebra, Suiza, $78 \mathrm{pp}$. 